\title{
Trajectory tracking for a serial robot manipulator passing through singular configurations based on the adaptive kinematics Jacobian method
}

\begin{abstract}
This paper discusses the use of artificial neural networks (ANNs) as a method of trajectory tracking control for a robotic system. Using an ANN does not require any prior knowledge of the kinematics model of the system being controlled; the basic idea of this concept is the use of the ANN to learn the characteristics of the robot system rather than to specify an explicit robot system model. In this approach, disadvantages of some schemes such as the fuzzy learning control, for example, have been elevated. Off-line training was performed for a geometric trajectory that is free of obstacles. Studying the kinematics Jacobian of serial manipulators by using ANNs has two problems: one of these is the selection of the appropriate configuration of the network and the other is the generation of a suitable training dataset. In this approach, although this is very difficult in practice, training data were recorded experimentally from sensors fixed on each joint to overcome the effect of kinematics uncertainties present in the real world such as ill-defined linkage parameters, links flexibility, and backlashes in the gear train. Then two network configurations were compared to find the best configuration to be used. Finally, the simulation results were verified experimentally using a general six-degree-of-freedom (DOF) serial robot manipulator.
\end{abstract}

Keyword: Artificial neural networks; Trajectory tracking; Kinematics; Robot control. 